\title{
Delay and poor diagnosis of Down syndrome in KwaZulu-Natal, South Africa: A retrospective review of postnatal cytogenetic testing
}

\author{
M Willoughby, ${ }^{1} \mathrm{MB} \mathrm{ChB}, \mathrm{DCH}(\mathrm{SA})$; C Aldous, ${ }^{2} \mathrm{PhD}$; M Patrick, ${ }^{2,3}$ MB ChB, DCH (SA), FCPaed (SA); S Kavonic, ${ }^{4}$ BSc Hons; \\ A Christianson, ${ }^{5}$ FRCP (Edin), MA \\ ${ }^{1}$ Department of Paediatrics and Child Health, School of Clinical Medicine, College of Health Sciences, Nelson R Mandela School of Medicine, \\ University of KwaZulu-Natal, Durban, South Africa \\ ${ }^{2}$ School of Clinical Medicine, College of Health Sciences, Nelson R Mandela School of Medicine, University of KwaZulu-Natal, Durban, \\ South Africa \\ ${ }^{3}$ Greys Hospital, Pietermaritzburg Metropolitan Hospitals Complex, South Africa \\ ${ }^{4}$ Tissue Immunology and Genetics, Specialised Laboratory Services Department, South African National Blood Service, Pinetown, KwaZulu- \\ Natal, South Africa \\ ${ }^{5}$ Wits Centre for Ethics, University of the Witwatersrand, Johannesburg, South Africa
}

Corresponding author: M Willoughby (markwillo@gmail.com)

\begin{abstract}
Background. Down syndrome (DS) is the most common chromosomal disorder in newborns. Until 20 years ago DS was considered rare in black African children in South Africa (SA). Lack of awareness of DS on the part of medical staff in SA, and difficulty in diagnosing it, appear to persist.

Objectives. To establish an epidemiological profile of DS and investigate the ability of clinicians in KwaZulu-Natal Province (KZN), SA, to make accurate clinical diagnoses of DS.

Methods. Records at the South African National Blood Service cytogenetic laboratory in Pinetown, KZN, were examined for all tests for clinically suspected DS undertaken during January 2009 - December 2013 and all cytogenetically proven DS test results. Age at diagnosis, the hospital from where the test was sent and type of chromosomal pattern for each confirmed DS test result were recorded.

Results. Of a total of 1578 tests requested, 875 confirmed DS, indicating that clinicians correctly clinically diagnosed DS $55.4 \%$ of the time. The average age of cytogenetic diagnosis of DS was 1 year and 20 days. The minimum population prevalence of DS was $0.8 / 1000$.

Conclusions. The diagnosis of DS is a challenge in KZN, potentiating missed opportunities for early intervention. The relatively low population prevalence of DS may be attributable to a lack of confirmatory cytogenetic tests or missed clinical diagnoses. It may also be attributable to a high mortality rate for children with DS in the province.
\end{abstract}

S Afr Med J 2016;106(6):626-629. DOI:10.7196/SAMJ.2016.v106i6.10105

Down syndrome (DS) is the most common chromosomal disorder in newborns, with a birth prevalence of approximately 2/1 000 live births in South Africa (SA). ${ }^{[1,2]}$ However, little is known about the epidemiological profile of this syndrome in KwaZulu-Natal Province (KZN). Before the 1980s DS was thought to be rare in black African population groups. ${ }^{[3]}$ In 1982, Adeyokunnu ${ }^{[4]}$ dispelled this myth in a retrospective study on the birth prevalence of DS in Ibadan, Nigeria, documenting a birth prevalence of $1.16 / 1000$ live births. ${ }^{[4]}$ It was not until the mid-1990s, when Venter et al. ${ }^{[5]}$ documented a birth prevalence of 2.1/1 000 live births in rural Limpopo Province and Kromberg et al. ${ }^{[6]}$ 1.8/1 000 live births at Chris Hani Baragwanath Hospital in Soweto, Johannesburg, that the high birth prevalence of DS was appreciated in SA. The high birth prevalence of DS in developing countries (2 - 3/1 000 live births) is attributed to the high proportion of pregnant woman who are of advanced maternal age (AMA) ${ }^{[2]}$ However, little further work has been undertaken on DS in black African children in SA, and Christianson's ${ }^{[3]}$ concerns about lack of awareness and difficulty in diagnosing DS by medical staff still appear to pertain. He described difficulties in recognising DS in black African neonates and young children, resulting in delays in diagnosing DS in SA, where only $16.4 \%$ of cases were diagnosed during the infant's postnatal stay in the hospital or clinic. ${ }^{[3]}$ The many abnormalities associated with
DS make early diagnosis important in the care of children with the disorder. ${ }^{[7]}$ Early detection of treatable disorders associated with DS, such as hypothyroidism, may prevent further irreversible mental and physical disabilities. ${ }^{[8]}$ The high cost of medical care for patients affected with $\mathrm{DS}^{[1]}$ also highlights the need for a largely preventive healthcare programme for these patients. Most of the improvements in quality of life for patients with DS are related to implementing early intervention programmes and improving support for parents. ${ }^{[7]}$ Effective preventive care benefits from early diagnosis of the disorder.

\section{Objectives}

To establish an epidemiological profile of postnatal DS in KZN and highlight the issues that arise therefrom, including indicating the burden of this congenital disorder in the province. The age of cytogenetic diagnosis of DS and the ratio of clinically suspected to cytogenetically proven DS were used as an indication of the abilities of clinicians in the province to diagnose DS accurately.

\section{Methods}

The log books and electronic records at the South African National Blood Service (SANBS) cytogenetic laboratory in Pinetown, KZN, were perused for postnatal chromosomal blood tests for clinically 
suspected and cytogenetically proven DS undertaken during the period January 2009 . December 2013.

The SANBS mainly serves the state sector in KZN and does all the medical genetic testing for DS for the state sector in the province. Blood samples are referred to the SANBS cytogenetic laboratory along with a document in which the referring clinician states the suspected disorder for which testing is being done. Data were collected onto an Excel spreadsheet. Age at diagnosis, hospital from which the blood sample was sent and the type of chromosomal pattern for each confirmed DS test result within the time period were recorded. To ensure anonymity, labelling of test results was resolved by number and patient's names were not recorded. Duplicate test results, where found, were excluded. Ethics approval to conduct the study was obtained from the University of KwaZulu-Natal Biomedical Research Ethics Committee (Ref. No. BE 431/14). Permission to use data from SANBS was granted before data collection began.

\section{Results}

From 1 January 2009 to 31 December 2013, blood samples from a total of 1578 patients suspected of having DS were sent to the laboratory for chromosomal confirmation of the diagnosis. Of these analyses, 875 confirmed the diagnosis of DS cytogenetically. The number of live births recorded for the same 5-year period in KZN was $1046623 .{ }^{[9]}$ This gives a minimum population prevalence of DS in the province of $0.8 / 1000(1 / 1205)$. The ratio of suspected to cytogenetically proven DS was $55.4 \%$ for this period. The number of tests for chromosomal confirmation of DS increased in each year of the study. However, the ratio of suspected to cytogenetically proven DS decreased over the same time period (Table 1).

During the study period, the number of negative tests when DS was clinically suspected was 703 . According to the 2009 2013 SANBS tariffs for blood chromosomal analysis, the total cost of these tests amounted to ZAR1 174 734. Chromosomal diagnoses of DS were made at an average age of 1 year and 20 days over the 5 -year period. Cytogenetic analyses revealed that $92.8 \%$ of the cases of DS were due to non-dysjunction, $5.0 \%$ involved translocation of chromosome 21 , and $2.2 \%$ were mosaic. Mosaic DS was diagnosed on average at 1 year 9 months, whereas translocation DS was diagnosed at a much earlier average age of 5 months and 6 days. Non-dysjunction DS was diagnosed at an average age of 1 year and 7 days (Table 2).

Fig. 1 illustrates the number of cytogenetically proven DS patients according to the healthcare facility category at which the initial diagnosis was made. Healthcare facilities were divided into specialist-driven facilities (academic/tertiary-level hospitals and regional/secondary-level hospitals) and non-specialist-driven facilities (district-level hospitals, primary healthcare hospitals and clinics). A very limited number of requests for DS diagnosis were received from private practice (private hospitals, private doctors' rooms and private pharmacies). This is probably because private laboratories carried out the tests for the private sector. Of all the cytogenetically proven DS patients, $76.7 \%$ $(n=671)$ were diagnosed in specialist-driven hospitals, which comprised 29.4\% $(n=15)$ of the healthcare facilities in KZN where initial diagnoses of DS were made. Nonspecialist hospitals and clinics constituted $56.9 \%(n=29)$ of the healthcare facilities at which diagnoses were made, but only $22.2 \%(n=194)$ of the total number of cytogenetically confirmed DS patients came from these sites. Private facilities constituted $13.7 \%(n=7)$ of all the healthcare facilities where diagnoses were made, and only $1.1 \%$ $(n=10)$ of cytogenetically confirmed DS patients came from those sources.

Fig. 2 illustrates the average age at which DS was cytogenetically confirmed according to the different healthcare facility categories. In specialist-driven facilities, 671 of DS patients (76.7\%) were correctly clinically diagnosed, at the lowest average age of 11 months and 15 days. In non-specialist-driven facilities, 194 DS patients $(22.2 \%)$ were diagnosed at a later average age of 1 year and 1 month. Only $10(1.1 \%)$ of DS patients were diagnosed in private facilities, at the latest average age of 7 years and 6 months.

Table 1. Ratios of clinically suspected DS to cytogenetically confirmed DS by year

\begin{tabular}{llllllll}
\hline & \multicolumn{5}{c}{ Year } & \\
\cline { 2 - 6 } & $\mathbf{2 0 0 9}$ & $\mathbf{2 0 1 0}$ & $\mathbf{2 0 1 1}$ & $\mathbf{2 0 1 2}$ & $\mathbf{2 0 1 3}$ & Total \\
\hline Suspected DS, $n$ & 230 & 280 & 355 & 321 & 392 & 1578 \\
Cytogenetically proven DS, $n$ & 154 & 171 & 203 & 169 & 178 & 875 \\
Correctly clinically diagnosed DS, \% & 67.0 & 61.0 & 57.2 & 52.6 & 45.4 & 55.4
\end{tabular}

Table 2. Proportions of the different types of DS with average age at cytogenetic diagnosis

\begin{tabular}{llll}
\hline & Non-dysjunction & Translocation & Mosaic \\
\hline Proportion, \% & 92.8 & 5.0 & 2.2 \\
Average age at diagnosis & $1 \mathrm{yr} 7 \mathrm{~d}$ & 5 mo 6 d & $1 \mathrm{yr} 9$ mo $14 \mathrm{~d}$
\end{tabular}

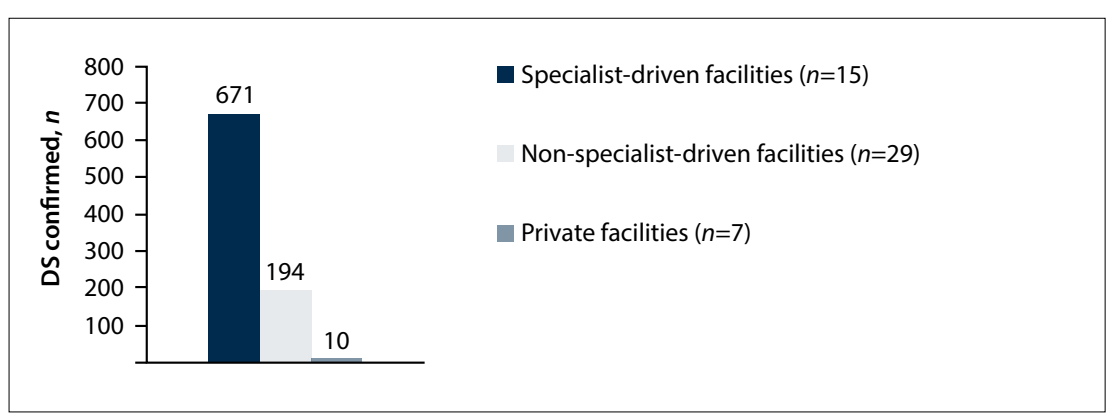

Fig. 1. Number of cytogenetically proven DS patients according to healthcare facility category.

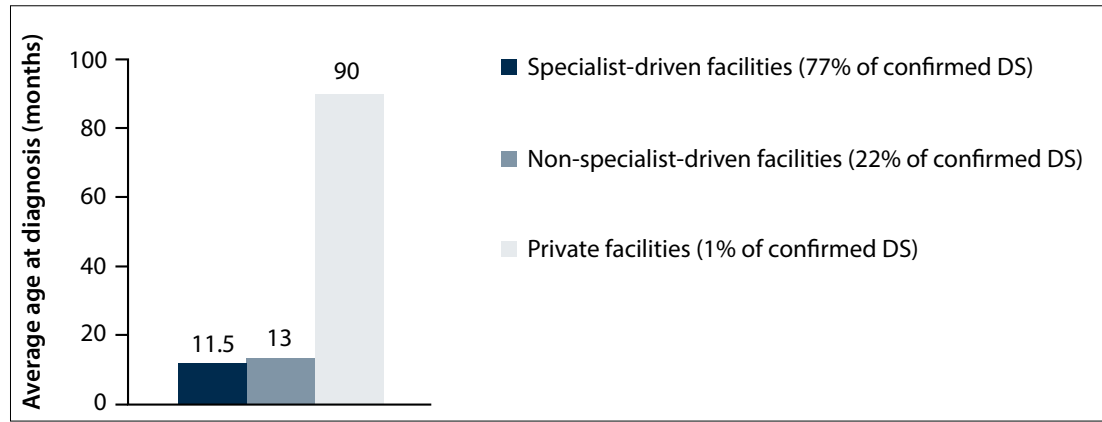

Fig. 2. Average age at diagnosis of DS according to healthcare facility category. 


\section{Discussion}

DS is a common congenital disorder that until 20 years ago was considered rare in black Africans of SA origin ${ }^{[3]}$ At that time it was shown that doctors, nurses and mothers lacked awareness of DS in black infants, that the disorder was easier to recognise in white infants, and that awareness of DS in communities was limited owing to a high mortality rate among affected infants, resulting in a low population prevalence of DS. ${ }^{[3,10]}$

DS is the most common chromosomal disorder in liveborn infants, with no predilection for race or socioeconomic group. It is the most well-known and well-researched genetic disorder, ${ }^{[10]}$ yet postnatal diagnosis in KZN, and in SA as a whole, remains a challenge. In KZN the average age at cytogenetic diagnosis of DS was 1 year and 20 days. In Gauteng and Limpopo provinces, more than half of the patients with DS attending a genetics clinic were diagnosed after 7 months of age. ${ }^{[3]}$ The diagnosis of DS is largely a clinical one, based on characteristic dysmorphic features, and may be confirmed by chromosomal testing. It is possible that the age of chromosomal diagnosis in this study is not an absolute indication of the age of clinical diagnosis, as some patients may have been correctly diagnosed and managed without chromosomal confirmation, or cytogenetic testing may have been done at a later stage. This may be due to concerns about the financial cost of obtaining a cytogenetic diagnosis. However, in our study clinicians clinically suspected DS accurately only $55 \%$ of the time. This differs markedly from a study in The Netherlands, where clinicians correctly clinically diagnosed DS $83 \%$ of the time. ${ }^{[11]}$ The average age of chromosomal diagnosis in that study was 1.8 days for infants born in hospital and 10.2 days for those born outside hospital. ${ }^{[1]}$ We postulate that the late age at which some children with DS were diagnosed in the private sector was because some patients' families did not receive the recognition and care that their children with DS deserved in public sector hospitals and therefore sought medical care elsewhere, resulting in delayed presentation to those facilities.

Diagnosis and prevention of DS with prenatal screening is not routinely and universally offered in public sector hospitals in SA. It is available in academic centres, but even in these, prenatal screening for DS using the most basic tool, a pregnant woman's age, is poorly undertaken. In a study of 48 women of AMA seen in academic hospitals in Johannesburg and having presented for antenatal care before 20 weeks' gestation, not one was offered counselling for prenatal diagnosis on the basis of her age. ${ }^{[12]}$ Only $6.6-8.6 \%$ of confirmed DS patients were diagnosed antenatally in Cape Town, where public health services are among the best developed in SA. ${ }^{[13]}$ The diagnosis of DS therefore remains almost entirely the responsibility of the healthcare workers who come into contact with these infants and children in the postnatal period. Congenital heart disease, which affects approximately half of all infants and children with DS, ${ }^{[11]}$ along with malnutrition and infections, is a significant cause of mortality for children with DS in SA, particularly in the context of limited availability of facilities for cardiac surgery ${ }^{[3]}$ In our study, the minimum population prevalence of DS was $0.8 / 1000$ over the 5 -year period. This is more than two times lower than birth prevalences of DS that were prospectively obtained in other areas of the country (2.1/1 000 live births in rural Limpopo ${ }^{[4]}$ and 1.8/1 000 in Soweto, Johannesburg $\left.{ }^{[5]}\right)$. In part this may be due to the poor ability of SA medical practitioners and nurses to diagnose DS. It may also reflect a falsely low calculated population prevalence because of some patients not being confirmed with chromosomal analysis, as described earlier. However, given the late age of diagnosis and the apparent inability to recognise DS accurately in KZN, it is surely contributed to by a high mortality rate among children with DS in the province. In an epidemiological study of intellectually disabled children aged 2 - 9 years in a rural community in Mpumalanga Province, the minimum population prevalence of DS was $0.75 / 1000$ and the authors calculated that two out of every three children with DS had died before the age of 2 years. ${ }^{[14]}$ It may be that in SA many children with DS are undiagnosed at the time of their death and recorded in under-5 mortality rates under various classifications indicative of DS symptoms or complications (e.g. pneumonia), resulting in the impact of DS as a public health issue being largely unrecognised.

\section{Conclusions}

Despite the limitations in using age of chromosomal diagnosis as an indication of age of clinical diagnosis, it is evident that medical practitioners and nursing staff in KZN have difficulty in making accurate clinical diagnoses of DS, resulting in missed opportunities for early intervention. With the proportion of confirmed diagnoses being poor to start with and then declining over time, it seems that the ability to diagnose DS clinically is waning. The cost of these clinically inappropriate chromosomal tests is considerable. The relatively low population prevalence of DS seen in this study, compared with far higher prospectively obtained birth prevalences in other areas of SA, suggests that many children with DS may in fact die before they are diagnosed. It is apparent from this study that, 20 years after showing that DS is a largely unrecognised problem in SA, little has changed, with potentially dire consequences for patients and their families. There is a great need to increase awareness of DS among medical and nursing staff and in communities, especially in hospitals and clinics that do not have specialist care and supervision. Our figures represent reliable data from the only cytogenetic service provider to the state sector in KZN. It is possible that similar studies in other provinces would reveal similar findings. In 2010 the World Health Organization's World Health Assembly passed resolution WHA63.17, urging member states to recognise congenital disorders (CDs) as a public health issue. They proposed that CDs would contribute to a failure to attain Millennium Development Goal 4 in low- and middle-income countries. ${ }^{[15]}$ If diagnosis, which is the first step in the clinical pathway to care for people with DS, the best-known and most common chromosomal disorder, is as poor as described in this study, what are the implications for children with other less easily recognisable CDs in KZN and SA? Malherbe et al. ${ }^{[16]}$ have recently suggested that the time is now optimal for the development of services for the care and prevention of congenital disorders in SA. The obvious place to start is with common disorders, especially DS.

Acknowledgements. The authors thank the SANBS Cytogenetics Laboratory in Pinetown for allowing access to their records and Nan Thuman for her work in extracting data from the SANBS computerised laboratory systems.

\section{References}

1. National Department of Health, South Africa. Human Genetics Policy Guidelines for the Management and Prevention of Genetic Disorders, Birth Defects and Disabilities. Pretoria: NDoH, 2000.

Christianson A, Howson CP, Modell B. March of Dimes: Global Report on Birth Defects, the Hidden Toll of Dying and Disabled Children. White Plains, NY: March of Dimes Birth Defects Foundation Toll of Dying and Disabled Children. White Plains, NY: March of Dimes Birth Defects Foundation,
2006:85. http://www.marchofdimes.org/materials/global-report-on-birth-defects-the-hidden-toll-ofdying-and-disabled-children-full-report.pdf (accessed 10 August 2015).

3. Christianson AL. Down syndrome in black South African infants and children - clinical features and delayed diagnosis. S Afr Med J 1997;87(8):992-995.

4. Adeyokunnu AA. The incidence of Down's syndrome in Nigeria. J Med Genet 1982:19(4):277-279.

5. Venter PA, Christianson AL, Hutamo CM, Makhura MP, Gericke GS. Congenital anomalies in rural black South African neonates - a silent epidemic? S Afr Med J 1995;85(1):11-15.

6. Kromberg JGR, Christianson AL, Duthie-Nurse G, Zwane E, Jenkins T. Down syndrome in the black population. S Afr Med J 1992;81(6):337-337.

Roizen NJ, Patterson D. Down's syndrome. Lancet 2003;361(9365):1281-1289. DOI:10.1016/S01406736(03)12987-X 
8. Moosa S, Segal DG, Christianson AL, Gregersen NE. Thyroid dysfunction in a cohort of South African children with Down syndrome. S Afr Med J 2013;103(12):966-970. DOI:10.7196/SAMJ.7111

9. Statistics South Africa. Statistical release P0305, Recorded live births 2013. http://www.statssa.gov.za/ publications/P0305/P03052013.pdf (accessed 14 July 2015).

publications/P0305/P03052013.pdf (accessed 14 July 2015). DOI:10.1136/jmg.33.2.89

11. Weijerman ME, van Furth AM, Vonk Noordegraaf A, van Wouwe JP, Broers CJM, Gemke RJBJ. Prevalence, neonatal characteristics, and first-year mortality of Down syndrome: A national study. Pediatr 2008;152(1):15-19. DOI:10.1016/j.jpeds.2007.09.045

12. Watcham S, Schön S, Christianson A. Neglect in the care of pregnant South African women of advanced maternal age. S Afr Med J 2007;97(1064):1068-1069

13. Urban M, Stewart C, Ruppelt T, Geerts L. Effectiveness of prenatal screening for Down syndrome on the basis of maternal age in Cape Town. S Afr Med J 2011;101(1):45-48.
14. Christianson A, Zwane M, Manga P, et al. Children with intellectual disability in rural South Africa: Prevalence and associated disability. J Intellect Disabil Res 2002;46(2):179-186. DOI:10.1046/j.13652788.2002.00390.

15. World Health Organization. Sixty-Third World Health Assembly - Birth Defects. Geneva: WHO, 2010 http://apps.who.int/gb/ebwha/pdf_files/WHA63/A63_R17-en.pdf (accessed 27 May 2015).

16. Malherbe HL, Christianson AL, Aldous C. Need for services for the care and prevention of congenital disorders in South Africa as the country's epidemiological transition evolves. S Afr Med J 015;105(3):186-188. DOI:10.7196/SAMJ.913

Accepted 16 March 2016 